\title{
Höhenakklimatisation und Kontaktlinsen
}

\section{Altitude adaptation and contact lenses}

\author{
Autor \\ Manfred Bufler \\ Institut \\ Kontaktlinsenstudio in einem Augenoptikfachbetrieb, Ruhpolding \\ Key words \\ altitude adaptation - lenses - corneal shape - meteorological \\ conditions \\ Bibliografie \\ DOI 10.1055/a-1149-6647 \\ Korrespondenzadresse \\ Manfred Bufler M.S. in clinical Optometry PCO PHD \\ in Optometry \\ Eisenbergstr. 1 a \\ 83324 Ruhpolding
}

\section{Retraction Notice}

Aufgrund eines technischen Fehlers wurde nicht die endgültige (autorisierte) Version veröffentlicht. Der Verlag entschuldigt sich für den Fehler.

Die autorisierte Version finden Sie unter:

https://doi.org/10.1055/s-0040-1722528

\section{ZUSAMMENFASSUNG}

Aufgrund der besonderen meteorologischen Bedingungen und den daraus folgenden Veränderungen der kornealen Form lassen sich Kontaktlinsen bis etwa 7000-7500 Hm noch einigermaßen ertragen. Besonders dünnen und sehr sauerstoffdurchlässigen weichen Silikonhydrogelkontaktlinsen sollte der Vorzug gegeben werden. Die dünnsten Kontaktlinsen gibt es bei den Tageslinsen. Kontaktlinsenflüssigkeiten und Kontaktlinsen sollten möglichst nahe am Körper transportiert werden. Die Brille und Desinfektionstücher gehören unbedingt in den Rucksack, ebenso wie künstliche Tränentropfen und die Sonnenbrille, die rund um das Auge gut abschließen sollte.

\section{ABSTRACT}

Extreme meteroligical conditions are expected on high altitude. That fact influences a extraordinary change of the corneal shape. So an endurable contact lens wearing is stated maximum up to an altitude of about $7000-7500 \mathrm{~m}$. High oxygen transmissable silicon hydrogel lenses should be prefered for this activities. The thinnest soft lenses are be found in the group of one day soft lenses. Generally, lenses and lens solutions should be carried near body on tour. Spectacles, desinfection tissues, artificial tears and tight fitted sun glasses are essential things having with you. 